\title{
Analysis of Timing Error Detectors for Orthogonal Space-Time Block Codes
}

\author{
Pawel A. Dmochowski, Peter J. McLane \\ Department of Electrical and Computer Engineering \\ Queen's University, Kingston, ON, K7L 3N6, Canada \\ Email:pdmochowski@ieee.org, mclanep@post.queensu.ca
}

\begin{abstract}
We analyze the properties of a class of low complexity timing error detectors for the purpose of timing error tracking in orthogonal space-time block coding receivers. For symmetrical signal constellations, under the assumptions of ideal data decisions and channel knowledge at the receiver, expressions for the S-curve, estimation error variance and the detector signal-to-noise ratio are derived. Simulations are used to confirm the analytical results and to evaluate the effects of data decision errors on the estimator properties. Symbolerror-rate performance is evaluated for a system operating in a frequency-flat Rayleigh fading environment, where the timing synchronization loss is found to be less than $0.3 \mathrm{~dB}$. In addition to receiver with perfect channel state information, results for pilotbased channel estimation are included in order to examine the effects of channel estimation errors on timing synchronization.
\end{abstract}

\section{INTRODUCTION}

The problem of parameter estimation, such as the timing epoch and the channel state, has long been recognized as a critical factor in the performance of multiple-input multipleoutput (MIMO) receivers. Timing acquisition in space-time coded modems was first addressed in [1], where the receiver obtains the timing information by maximizing the oversampled log-likelihood function derived from an orthogonal training sequence. A modification was proposed in [2], where the resulting algorithm significantly reduces the oversampling required to achieve a given mean squared error. More recently, Rajawat et al. [3] achieved a further reduction down to two samples per symbol by exploiting the pulse-shape information at the receiver.

In contrast to the coarse timing acquisition methods of [1][3], this paper focuses on the problem of low complexity timing error estimation for the purpose of timing error tracking. Previous work in [4] described a method for the design of timing error detectors (TEDs) for arbitrary orthogonal space-time block codes (OSTBCs), where the conditions for a difference of threshold crossings timing error measurements independent of channel fading were derived. This paper derives analytical expressions for the estimation error variance and output signalto-noise ratio (SNR) of the TEDs in [4] under the conditions of perfect channel knowledge and data decisions. The effects of data decision and channel estimation errors are examined by means of simulations.

The paper begins with a system overview in Section II. Section III presents a summary of the TED design process followed by the derivation of TED estimation variance and output SNR. Simulation results are given in Section IV, and we conclude with a summary of results in Section V.

\section{System OVERVIEW}

Consider an OSTBC communication system with $N_{t}$ transmit and $N_{r}$ receive antennas. The transmitter encodes $N_{s}$ information symbols and transmits them over $N_{t}$ antennas in $N_{c}$ time slots, resulting in a code rate of $R=N_{s} / N_{c}$. We denote the $l$ th $N_{t} \times N_{c}$ code block by $\mathbf{X}_{l}$, where boldface notation is used for matrices, and its $(i k)$ th entry by $x_{i}\left(l N_{c}+k\right)$. Note that $l$ is the block index, $k=0, \ldots, N_{c}-1$ is the time slot index within the block and $i=1, \ldots, N_{t}$ is the transmit antenna index. Let the $m$ th information symbol encoding block $\mathbf{X}_{l}$ be $a_{m}^{l}$, where $m=0, \ldots, N_{s}-1$. Then, $\mathbf{X}_{l}$ is given by [5]

$$
\mathbf{X}_{l}=\sum_{m=0}^{N_{s}-1} \Re\left\{a_{m}^{l}\right\} \mathbf{A}_{m}+i \Im\left\{a_{m}^{l}\right\} \mathbf{B}_{m},
$$

where the operators $\Re\{\cdot\}$ and $\Im\{\cdot\}$ return the real and imaginary parts of their arguments, respectively, and $\mathbf{A}_{m}$ and $\mathbf{B}_{m}$ are integer code matrices of dimension $N_{t} \times N_{c}$. The pulse shaping is split between the transmitter and the receiver, each employing a root raised cosine (RRC) filter denoted by $\tilde{g}(t)$. The combined Nyquist raised cosine pulse is represented by $g(t)=\tilde{g}(t) * \tilde{g}(t)$, where $*$ denotes convolution. We assume a frequency-flat Rayleigh fading channel modeled by a $N_{r} \times N_{t}$ matrix $\mathbf{H}$. It's components, denoted by $h_{j i}$, correspond to the state of the fading channel from $i$ th transmit to $j$ th receive antenna and are assumed to be independent and identically distributed (iid) for all $i$ and $j$ with a $\mathrm{U}$-shaped power spectrum of isotropic scattering and maximum Doppler frequency of $f_{D}$, assumed to be known.

The receiver diagram is given in Fig. 1. The received signal

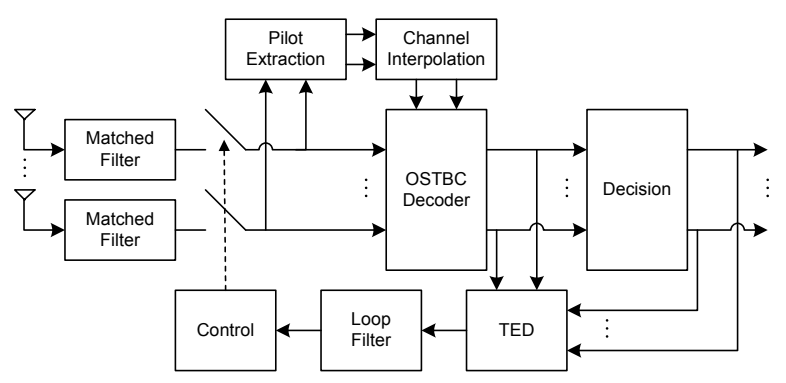

Fig. 1. Receiver Diagram. 
at antenna $j$ is given by ${ }^{1}$

$$
r_{j}(t)=\sum_{i=1}^{N_{t}} h_{j i}(t) \sum_{n^{\prime}} x_{i}\left[n^{\prime}\right] \tilde{g}\left(t-n^{\prime} T\right)+\tilde{\eta}_{j}(t),
$$

where $x_{i}\left[n^{\prime}\right]$ is the encoded symbol transmitted by antenna $i$ at time $n^{\prime}=l N_{c}+k$ and $\tilde{\eta}_{j}(t)$ is a zero mean complex Gaussian noise with variance $\sigma_{\tilde{\eta}}^{2}=N_{0} / 2$ per signal dimension. After matched filtering, the signal $y_{j}(t)=r_{j}(t) * \tilde{g}(t)$ is sampled at time instants $t_{n}=n T+\epsilon$, where $\epsilon$ is the unknown timing error, assumed to be equal on all branches. We express $\epsilon$ by $\epsilon=\tau-\hat{\tau}$ where $\tau$ is the timing offset at the receiver and $\hat{\tau}$ is the timing correction applied by the timing synchronization algorithm. Assuming the channel fading is sufficiently slow, such that $h_{j i}\left(t_{n}\right) \approx h_{j i}(n T) \triangleq h_{j i}[n]$, we can write

$$
y_{j}[n]=\sum_{i=1}^{N_{t}} h_{j i}[n] \sum_{n^{\prime}} x_{i}\left[n^{\prime}\right] g\left(n T-n^{\prime} T+\epsilon\right)+\eta_{j}[n],
$$

where $\eta_{j}[n]$ are the samples of the filtered noise $\eta_{j}(t)=\tilde{\eta}_{j}(t) *$ $\tilde{g}(t)$, which are uncorrelated if sampled at symbol rate.

Consider the output samples for $n=l N_{c}, \ldots,(l+1) N_{c}-1$, corresponding to time slots $k=0, \ldots, N_{c}-1$ within a code block $l$. We assume that the timing error $\epsilon$ is constant for the duration of one block. Similarly, we assume quasi-static fading, where $h_{j i}\left[l N_{c}\right] \approx h_{j i}\left[(l+1) N_{c}-1\right] \triangleq h_{j i}[l]$. Then, the output can be expressed by a $N_{r} \times N_{c}$ matrix $\mathbf{Y}_{l}$ [4]

$$
\mathbf{Y}_{l}=\mathbf{H}_{l} \sum_{n} \mathbf{X}_{l+n} \mathbf{G}_{\epsilon, n}+\mathbf{N}_{l}
$$

where $\mathbf{H}_{l}$ and $\mathbf{N}_{l}$ denote the channel state and noise matrices, respectively, and $\mathbf{G}_{\epsilon, n}$ is a $N_{c} \times N_{c}$ Toeplitz matrix given by

$$
\mathbf{G}_{\epsilon, n}=\left[\begin{array}{cccc}
g_{-n N_{c}}^{\epsilon} & g_{-n N_{c}+1}^{\epsilon} & g_{-n N_{c}+2}^{\epsilon} & \cdots \\
g_{-n N_{c}-1}^{\epsilon} & g_{-n N_{c}}^{\epsilon} & g_{-n N_{c}+1}^{\epsilon} & \ddots \\
g_{-n N_{c}-2}^{\epsilon} & g_{-n N_{c}-1}^{\epsilon} & g_{-n N_{c}}^{\epsilon} & \ddots \\
\vdots & \ddots & \ddots & \ddots
\end{array}\right],
$$

where we denote the pulse shape samples by $g_{n}^{\epsilon} \triangleq g(n T+\epsilon)$.

Finally, the detection variables for each information symbol $m=0, \ldots, N_{s}-1$ within a block $l$ are given by [5]

$$
s_{m}^{l}=\left\|\mathbf{H}_{l}\right\|^{-2}\left(\Re\left\{\operatorname{tr}\left(\mathbf{Y}_{l}^{H} \mathbf{H}_{l} \mathbf{A}_{m}\right)\right\}-i \Im\left\{\operatorname{tr}\left(\mathbf{Y}_{l}^{H} \mathbf{H}_{l} \mathbf{B}_{m}\right)\right\}\right),
$$

where $\mathbf{A}_{m}$ and $\mathbf{B}_{m}$ are the encoding matrices used in (1), $\operatorname{tr}(\cdot)$ denotes the trace operator, superscript $H$ is the Hermitian transpose and $\left\|\mathbf{H}_{l}\right\|$ is the Frobenius norm of $\mathbf{H}_{l}$. While (6) represents Maximum Likelihood (ML) detection when no timing error is present, we assume that in the case of timing error tracking, where $|\epsilon|<<1$, the above expression is a close approximation to ML detection. The projection of $s_{m}^{l}$ onto the signal constellation forms the data decisions $\hat{a}_{m}^{l}$.

\footnotetext{
${ }^{1}$ We differentiate between discrete and continuous quantities by placing their arguments within square brackets and parenthesis, respectively, e.g., $x(t)$ or $x[n]=x(n T)$.
}

\section{TED FOR OSTBC}

In order to maintain compact notation, especially in the Appendix, we denote the expectation conditioned on $\mathbf{H}$ by $E^{\mathbf{H}}\{\cdot\}$, while the expectation taken over $\mathbf{H}$ will be denoted by $E_{\mathbf{H}}\{\cdot\}$. Expectation over all random variables is thus given by $E\{\cdot\}=E_{\mathbf{H}}\left\{E^{\mathbf{H}}\{\cdot\}\right\}$. In the sequel, $E_{\mathbf{H}}\{\cdot\}$ is always computed by simulation as the argument is too complex for analysis.

\section{A. TED S-Curve}

We consider a general expression for a TED in the form of a linear combination of products $a_{m} s_{m}$ and $a_{m}^{*} s_{m}$, that is

$$
\hat{\epsilon}=\Re\left(\sum_{k} \alpha_{k} a_{n_{\alpha, k}} s_{m_{\alpha, k}}+\beta_{k} a_{n_{\beta, k}}^{*} s_{m_{\beta, k}}\right)
$$

and define a parameter set containing sum weights as well as the indices of the data symbols and decision variables in (7), as

$$
\mathcal{S}=\left\{\alpha_{k}, \beta_{k}, m_{\alpha, k}, n_{\alpha, k}, m_{\beta, k}, n_{\beta, k}\right\}
$$

For notational simplicity we have dropped the block index $l$ with the understanding that all quantities refer to a code block $l$. The S-curve, that is the expectation, taken over the data symbols, channel state and noise, of the estimator in (7), can be obtained by first computing the expectation conditioned on the channel variable $\mathbf{H}$ followed by the expectation over $\mathbf{H}$. It has been shown in [4] that for the general form of the estimator in (7),

$$
E^{\mathbf{H}}\{\hat{\epsilon}\}=\rho_{2}\|\mathbf{H}\|^{-2} \operatorname{tr}\left\{\boldsymbol{\Gamma} \Re\left(\mathbf{H}^{H} \mathbf{H}\right)\right\},
$$

where, using superscripts $R$ and $I$ to denote real and imaginary components, we define a constellation-dependent constant

$$
\rho_{p} \triangleq E\left\{\left(a_{i}^{R}\right)^{p}\right\}=E\left\{\left(a_{i}^{I}\right)^{p}\right\} .
$$

We have assumed a symmetric constellation in the second equality of (10). Matrix $\Gamma$ in (9), which is dependent on the coefficient set $\mathcal{S}$ chosen in (7), is given by [4]

$$
\begin{array}{r}
\boldsymbol{\Gamma}=\sum_{k}\left\{\alpha_{k}\left(\mathbf{A}_{m_{\alpha, k}} \mathbf{G}_{\epsilon}^{H} \mathbf{A}_{n_{\alpha, k}}^{H}-\mathbf{B}_{m_{\alpha, k}} \mathbf{G}_{\epsilon}^{H} \mathbf{B}_{n_{\alpha, k}}^{H}\right)+\right. \\
\left.\beta_{k}\left(\mathbf{A}_{m_{\beta, k}} \mathbf{G}_{\epsilon}^{H} \mathbf{A}_{n_{\beta, k}}^{H}+\mathbf{B}_{m_{\beta, k}} \mathbf{G}_{\epsilon}^{H} \mathbf{B}_{n_{\beta, k}}^{H}\right)\right\},
\end{array}
$$

where we let $\mathbf{G}_{\epsilon}$ represent $\mathbf{G}_{\epsilon, n}$ for $n=0$. It was shown in [4] that if $\boldsymbol{\Gamma}$ satisfies

$$
\boldsymbol{\Gamma}=f\left(\mathbf{G}_{\epsilon}\right) \mathbf{I}+\mathbf{D},
$$

where

1) $f\left(\mathbf{G}_{\epsilon}\right)$ is a scalar function of $\mathbf{G}_{\epsilon}$ that returns a timing error measurement (TEM) in the form of a difference of threshold crossings

2) $\mathbf{D}$ is an antisymmetric matrix,

then from (7)-(11),

$$
E^{\mathbf{H}}\{\hat{\epsilon}\}=\mu+\delta_{\hat{\epsilon}},
$$

where $\mu=\rho_{2} f\left(\mathbf{G}_{\epsilon}\right)$ and $\delta_{\hat{\epsilon}}$, which is dependent on $\mathbf{H}$, will be referred to as the TEM bias. If conditions 1) and 2) are 
satisfied, $\delta_{\hat{\epsilon}}=0$ [4], resulting in a robust TED. If only condition 1) is satisfied, that is $\mathbf{D}$ is a matrix with zeros on the main diagonal, then [4]

$$
\delta_{\hat{\epsilon}}=\rho\|\mathbf{H}\|^{-2} \sum_{m=1}^{N_{t}} \sum_{\substack{i=1 \\ i \neq m}}^{N_{t}} \sum_{j=1}^{N_{r}} d_{m i} \Re\left(h_{j i}^{*} h_{j m}\right),
$$

where $d_{m i}$ denotes the $(m, i)$ th entry of D. For iid channels, the expectation over $\mathbf{H}$ of the numerator in (14) is zero, and thus due to the presence of the channel norm in the denominator, the effect of the bias is small. We refer to such a TED as quasi-robust. Finally, the TED S-curve is obtained from (13) by computing the expectation over the channel fading matrix $\mathbf{H}$, that is

$$
E\{\hat{\epsilon}\}=\mu+\bar{\delta}_{\hat{\epsilon}}
$$

where the expectation of the bias $\bar{\delta}_{\hat{\epsilon}}=E_{\mathbf{H}}\left\{\delta_{\hat{\epsilon}}\right\}$ must be evaluated by simulation.

\section{B. TED Variance and Output SNR}

In this section we derive the variance of the timing error estimate produced by the TEDs described in Section III-A. The solution, together with the expression for the S-curve in (15) allows us to obtain the output SNR of the detector.

The variance of the TED output is defined by

$$
\sigma_{\hat{\epsilon}}^{2}=E\left\{\hat{\epsilon}^{2}\right\}-[E\{\hat{\epsilon}\}]^{2} \text {. }
$$

Similarly to the approach taken in Section III-A, we evaluate the expectations conditioned on $\mathbf{H}$, followed by the average over the channel fading variable. By examining (7), one notes that the solution to $E^{\mathbf{H}}\left\{\hat{\epsilon}^{2}\right\}$ for various TED formulations can be obtained by considering $E^{\mathbf{H}}\left\{a_{i}^{R} a_{j}^{R} s_{m}^{R} s_{n}^{R}\right\}, E\left\{a_{i}^{I} a_{j}^{I} s_{m}^{I} s_{n}^{I}\right\}$ and $E^{\mathbf{H}}\left\{a_{i}^{R} a_{j}^{I} s_{m}^{R} s_{n}^{I}\right\}$. We begin with $E^{\mathbf{H}}\left\{a_{i}^{R} a_{j}^{R} s_{m}^{R} s_{n}^{R}\right\}$, which from (6) can be expressed as

$$
\begin{aligned}
E^{\mathbf{H}}\left\{a_{i}^{R} a_{j}^{R} s_{m}^{R} s_{n}^{R}\right\}=\|\mathbf{H}\|^{-4} E^{\mathbf{H}}\left\{a_{i}^{R} a_{j}^{R} \times\right. \\
\left.\operatorname{tr}\left\{\mathbf{A}_{m} \Re\left(\mathbf{Y}^{H} \mathbf{H}\right)\right\} \operatorname{tr}\left\{\mathbf{A}_{n} \Re\left(\mathbf{Y}^{H} \mathbf{H}\right)\right\}\right\},
\end{aligned}
$$

where we used the fact that $\Re(\operatorname{tr}(\cdot))=\operatorname{tr}(\Re(\cdot)), \operatorname{tr}(\mathbf{A B})=$ $\operatorname{tr}(\mathbf{B A})$ and that $\mathbf{A}_{m}$ and $\mathbf{A}_{n}$ are real valued. Assuming data is drawn from a symmetrical constellation, and that data and noise are independent from each other, the solution to (17), details of which are presented in the Appendix, is given by

$$
E^{\mathbf{H}}\left\{a_{i}^{R} a_{j}^{R} s_{m}^{R} s_{n}^{R}\right\}=\|\mathbf{H}\|^{-4} \operatorname{tr}\left\{\rho_{2}^{2} \boldsymbol{\Phi}_{i j m n}^{R R}+\rho_{2} \frac{N_{0}}{2} \Delta_{i j m n}^{R R}\right\},
$$

where $\boldsymbol{\Phi}_{i j m n}^{R R}$ is given by (19) and

$$
\boldsymbol{\Delta}_{i j m n}^{R R}= \begin{cases}\left(\mathbf{A}_{m} \otimes \mathbf{A}_{n}\right) \boldsymbol{\Lambda}_{N}\left(\boldsymbol{\Omega}_{\mathrm{RR}}^{\prime}+\boldsymbol{\Omega}_{\mathrm{II}}^{\prime}\right) & i=j \\ 0 & i \neq j,\end{cases}
$$

with $\otimes$ denoting the Kronecker matrix product and where the $N_{c} N_{c} \times N_{r} N_{r}$ matrix $\Lambda_{N}$ is given by

$$
\boldsymbol{\Lambda}_{N}(i, j)= \begin{cases}1 & i=n N_{r}+n+1, j=m N_{c}+m+1 \\ 0 & \text { elsewhere }\end{cases}
$$

for $n=0, \ldots, N_{r}-1$ and $m=0, \ldots, N_{c}-1$. The constant $\rho_{p}^{\prime}$ in (19) is given by

$$
\rho_{p}^{\prime} \triangleq E\left\{\left(a_{i}^{R}\right)^{p}\left(a_{i}^{I}\right)^{p}\right\} .
$$

In (19) and (20) we have defined channel dependent variables $\boldsymbol{\Omega}_{\mathrm{RR}}=\Re\left(\mathbf{H}^{H} \mathbf{H}\right) \otimes \Re\left(\mathbf{H}^{H} \mathbf{H}\right) \quad \boldsymbol{\Omega}_{\mathrm{II}}=\Im\left(\mathbf{H}^{H} \mathbf{H}\right) \otimes \Im\left(\mathbf{H}^{H} \mathbf{H}\right)$ $\boldsymbol{\Omega}_{\mathrm{RI}}=\Re\left(\mathbf{H}^{H} \mathbf{H}\right) \otimes \Im\left(\mathbf{H}^{H} \mathbf{H}\right) \quad \boldsymbol{\Omega}_{\mathrm{IR}}=\Im\left(\mathbf{H}^{H} \mathbf{H}\right) \otimes \Re\left(\mathbf{H}^{H} \mathbf{H}\right)$

and

$$
\begin{array}{ll}
\boldsymbol{\Omega}_{\mathrm{RR}}^{\prime}=\Re(\mathbf{H}) \otimes \Re(\mathbf{H}) & \boldsymbol{\Omega}_{\mathrm{II}}^{\prime}=\Im(\mathbf{H}) \otimes \Im(\mathbf{H}) \\
\boldsymbol{\Omega}_{\mathrm{RI}}^{\prime}=\Re(\mathbf{H}) \otimes \Im(\mathbf{H}) & \boldsymbol{\Omega}_{\mathrm{IR}}^{\prime}=\Im(\mathbf{H}) \otimes \Re(\mathbf{H}) .
\end{array}
$$

The solution to $E^{\mathbf{H}}\left\{a_{i}^{I} a_{j}^{I} s_{m}^{I} s_{n}^{I}\right\}$ can be obtained by following the same methodology as for $E^{\mathbf{H}}\left\{a_{i}^{R} a_{j}^{R} s_{m}^{R} s_{n}^{R}\right\}$. The details are once again shown in the Appendix, with the solution given by

$$
E^{\mathbf{H}}\left\{a_{i}^{I} a_{j}^{I} s_{m}^{I} s_{n}^{I}\right\}=\|\mathbf{H}\|^{-4} \operatorname{tr}\left\{\rho_{2}^{2} \boldsymbol{\Phi}_{i j m n}^{I I}+\rho_{2} \frac{N_{0}}{2} \boldsymbol{\Delta}_{i j m n}^{I I}\right\},
$$

with $\boldsymbol{\Phi}_{i j m n}^{I I}$ given by (26) and

$$
\boldsymbol{\Delta}_{i j m n}^{I I}= \begin{cases}\left(\mathbf{B}_{m} \otimes \mathbf{B}_{n}\right) \boldsymbol{\Lambda}_{N}\left(\boldsymbol{\Omega}_{\mathrm{RR}}^{\prime}+\boldsymbol{\Omega}_{\mathrm{II}}^{\prime}\right) & i=j \\ 0 & i \neq j,\end{cases}
$$

where $\boldsymbol{\Lambda}_{N}$ is given by (21).

Finally, the solution to $E^{\mathbf{H}}\left\{a_{i}^{R} a_{j}^{I} s_{m}^{R} s_{n}^{I}\right\}$, as outlined in the Appendix, is given by

$$
E^{\mathbf{H}}\left\{a_{i}^{R} a_{j}^{I} s_{m}^{R} s_{n}^{I}\right\}=\|\mathbf{H}\|^{-4} \operatorname{tr}\left\{\tilde{\rho} \boldsymbol{\Phi}_{i j m n}^{R I}\right\},
$$

where

$$
\begin{aligned}
\boldsymbol{\Phi}_{i j m n}^{R I}= & \left(\mathbf{A}_{m} \mathbf{G}_{\epsilon, 0}^{H} \otimes \mathbf{B}_{n} \mathbf{G}_{\epsilon, 0}^{H}\right) \times \\
& \left(\left(\mathbf{A}_{i}^{H} \otimes \mathbf{B}_{j}^{H}\right) \boldsymbol{\Omega}_{\mathrm{RR}}-\left(\mathbf{B}_{j}^{H} \otimes \mathbf{A}_{i}^{H}\right) \boldsymbol{\Omega}_{\mathrm{II}}\right)
\end{aligned}
$$

and $\tilde{\rho}$ is defined as

$$
\tilde{\rho}= \begin{cases}\rho_{2}^{2} & i \neq j \\ \rho_{2}^{\prime} & i=j\end{cases}
$$

Using (18), (25) and (28), one can obtain $E\left\{\hat{\epsilon}^{2}\right\}$ for a particular TED, that is

$$
E\left\{\hat{\epsilon}^{2}\right\}=E_{\mathbf{H}}\left\{\|\mathbf{H}\|^{-4} \operatorname{tr}\left\{\rho_{2}^{2} \boldsymbol{\Sigma}_{\boldsymbol{\Phi}}+\rho_{2} \frac{N_{0}}{2} \boldsymbol{\Sigma}_{\boldsymbol{\Delta}}\right\}\right\},
$$

where $\boldsymbol{\Sigma}_{\boldsymbol{\Phi}}$ and $\boldsymbol{\Sigma}_{\boldsymbol{\Delta}}$ correspond to the linear combination of $\boldsymbol{\Phi}_{i j m n}^{R R}, \boldsymbol{\Phi}_{i j m n}^{I I}, \boldsymbol{\Phi}_{i j m n}^{R I}$ and $\boldsymbol{\Delta}_{i j m n}^{R R}, \boldsymbol{\Delta}_{i j m n}^{I I}$, respectively, as determined by the polynomial expansion in $E\left\{\hat{\epsilon}^{2}\right\}$ for a particular TED. Due to the complexity of the expressions involved, the expectation over $\mathbf{H}$ must once again be carried out numerically, as will be done in Section III-D for specific examples of TEDs.

Finally, we will define the TED SNR by the square of the TEM component of (15) divided by the equivalent noise power, that is

$$
\begin{aligned}
S N R_{T E D} & =\frac{\mu^{2}}{E\left\{(\hat{\epsilon}-\mu)^{2}\right\}} \\
& =\frac{\mu^{2}}{\sigma_{\hat{\epsilon}}^{2}+\bar{\delta}_{\hat{\epsilon}}^{2}}
\end{aligned}
$$




$$
\begin{gathered}
\mathbf{\Phi}_{i j m n}^{R R}= \begin{cases}\left(\mathbf{A}_{m} \mathbf{G}_{\epsilon, 0}^{H} \otimes \mathbf{A}_{n} \mathbf{G}_{\epsilon, 0}^{H}\right)\left(\mathbf{A}_{j}^{H} \otimes \mathbf{A}_{i}^{H}+\mathbf{A}_{i}^{H} \otimes \mathbf{A}_{j}^{H}\right) \boldsymbol{\Omega}_{R R} & i \neq j \\
\left(\mathbf{A}_{m} \mathbf{G}_{\epsilon, 0}^{H} \otimes \mathbf{A}_{n} \mathbf{G}_{\epsilon, 0}^{H}\right)\left[\left(\frac{\rho_{4}}{\rho_{2}^{2}}-1\right)\left(\mathbf{A}_{i}^{H} \otimes \mathbf{A}_{i}^{H}\right) \boldsymbol{\Omega}_{R R}+\left(\frac{\rho_{2}^{\prime}}{\rho_{2}^{2}}-1\right)\left(\mathbf{B}_{i}^{H} \otimes \mathbf{B}_{i}^{H}\right) \boldsymbol{\Omega}_{\mathrm{II}}\right] & \\
+\sum_{l} \sum_{k=0}^{N_{s}-1}\left(\mathbf{A}_{m} \mathbf{G}_{\epsilon, l}^{H} \otimes \mathbf{A}_{n} \mathbf{G}_{\epsilon, l}^{H}\right)\left[\left(\mathbf{A}_{k}^{H} \otimes \mathbf{A}_{k}^{H}\right) \boldsymbol{\Omega}_{R R}+\left(\mathbf{B}_{k}^{H} \otimes \mathbf{B}_{k}^{H}\right) \boldsymbol{\Omega}_{\mathrm{II}}\right] & i=j\end{cases} \\
\mathbf{\Phi}_{i j m n}^{I I}= \begin{cases}\left(\mathbf{B}_{m} \mathbf{G}_{\epsilon, 0}^{H} \otimes \mathbf{B}_{n} \mathbf{G}_{\epsilon, 0}^{H}\right)\left(\mathbf{B}_{j}^{H} \otimes \mathbf{B}_{i}^{H}+\mathbf{B}_{i}^{H} \otimes \mathbf{B}_{j}^{H}\right) \boldsymbol{\Omega}_{R R} & i \neq j \\
\left(\mathbf{B}_{m} \mathbf{G}_{\epsilon, 0}^{H} \otimes \mathbf{B}_{n} \mathbf{G}_{\epsilon, 0}^{H}\right)\left[\left(\frac{\rho 4}{\rho_{2}^{2}}-1\right)\left(\mathbf{B}_{i}^{H} \otimes \mathbf{B}_{i}^{H}\right) \boldsymbol{\Omega}_{R R}+\left(\frac{\rho_{2}^{\prime}}{\rho_{2}^{2}}-1\right)\left(\mathbf{A}_{i}^{H} \otimes \mathbf{A}_{i}^{H}\right) \boldsymbol{\Omega}_{\mathrm{II}}\right] & \\
+\sum_{l} \sum_{k=0}^{N_{s}-1}\left(\mathbf{B}_{m} \mathbf{G}_{\epsilon, l}^{H} \otimes \mathbf{B}_{n} \mathbf{G}_{\epsilon, l}^{H}\right)\left[\left(\mathbf{B}_{k}^{H} \otimes \mathbf{B}_{k}^{H}\right) \boldsymbol{\Omega}_{R R}+\left(\mathbf{A}_{k}^{H} \otimes \mathbf{A}_{k}^{H}\right) \boldsymbol{\Omega}_{\mathrm{II}}\right] & i=j\end{cases}
\end{gathered}
$$

where the second equality is obtained using (15) and (16). Furthermore, using the fact that the effect of the bias is small, as discussed in III-A, we can approximate (32) by

$$
S N R_{T E D} \approx \frac{[E\{\hat{\epsilon}\}]^{2}}{\sigma_{\hat{\epsilon}}^{2}} .
$$

\section{Examples of TEDs}

As proposed in [6], a TED for 2-transmit antenna OSTBC (Alamouti encoding) has the form of

$$
\hat{\epsilon}_{(2)}=\Re\left\{a_{0} s_{1}-a_{1} s_{0}\right\}=a_{1}^{R} s_{0}^{R}-a_{1}^{I} s_{0}^{I}-a_{0}^{R} s_{1}^{R}+a_{0}^{I} s_{1}^{I} .
$$

For this case, the matrix $\Gamma$ can be shown to be

$$
\boldsymbol{\Gamma}_{(2)}=2\left[\begin{array}{cc}
g_{-1}^{\epsilon}-g_{1}^{\epsilon} & 0 \\
0 & g_{-1}^{\epsilon}-g_{1}^{\epsilon}
\end{array}\right],
$$

that is, $f\left(\boldsymbol{G}_{\epsilon}\right)=2\left(g_{-1}^{\epsilon}-g_{1}^{\epsilon}\right)$, and $\boldsymbol{D}=0$. Thus, the S-curve of the TED in (34) is given by

$$
E\left\{\hat{\epsilon}_{(2)}\right\}=\mu=2 \rho_{2}\left(g_{-1}^{\epsilon}-g_{1}^{\epsilon}\right),
$$

with $\delta_{\hat{\epsilon}_{(2)}}=0$, resulting in a robust timing estimate. The variance for the TED in (34) can be calculated using (16) and (31), where

$$
\begin{gathered}
\boldsymbol{\Sigma}_{\boldsymbol{\Phi}}=\boldsymbol{\Phi}_{1100}^{R R}+\boldsymbol{\Phi}_{0011}^{R R}-2 \boldsymbol{\Phi}_{1010}^{R R}+\boldsymbol{\Phi}_{1100}^{I I}+\boldsymbol{\Phi}_{0011}^{I I} \\
-2 \boldsymbol{\Phi}_{1010}^{I I}-2 \boldsymbol{\Phi}_{1100}^{R I}-2 \boldsymbol{\Phi}_{0011}^{R I}+2 \boldsymbol{\Phi}_{1001}^{R I}+2 \boldsymbol{\Phi}_{0110}^{R I},
\end{gathered}
$$

and

$$
\boldsymbol{\Sigma}_{\boldsymbol{\Delta}}=\boldsymbol{\Delta}_{1100}^{R R}+\boldsymbol{\Delta}_{0011}^{R R}+\boldsymbol{\Delta}_{1100}^{I I}+\boldsymbol{\Delta}_{0011}^{I I} .
$$

A number of higher order OSTBC encoders have been presented in literature [7], [8]. As an example we consider an $N_{t}=4$ code given from [8], given by

$$
\mathbf{X}_{(4)}=\left[\begin{array}{cccc}
a_{0} & a_{1}^{*} & a_{2}^{*} & 0 \\
-a_{1} & a_{0}^{*} & 0 & -a_{2}^{*} \\
-a_{2} & 0 & a_{0}^{*} & a_{1}^{*} \\
0 & a_{2} & -a_{1} & a_{0}
\end{array}\right],
$$

for which a TED was proposed in [4], in the form of

$$
\hat{\epsilon}_{(4)}=\Re\left\{a_{1} s_{0}-a_{0} s_{1}+a_{1}^{*} s_{0}-a_{0}^{*} s_{1}\right\}=2\left(a_{1}^{R} s_{0}^{R}-a_{0}^{R} s_{1}^{R}\right) .
$$

The S-curve is given by [4]

$$
E\left\{\hat{\epsilon}_{(4)}\right\}=2 \rho_{2}\left(g_{-1}^{\epsilon}-g_{1}^{\epsilon}\right)+\bar{\delta}_{\hat{\epsilon}_{(4)}},
$$

that is $\mu=2 \rho_{2}\left(g_{-1}^{\epsilon}-g_{1}^{\epsilon}\right)$ and $\bar{\delta}_{\hat{\epsilon}_{(4)}}=E_{\mathbf{H}}\left\{\delta_{\hat{\epsilon}_{(4)}}\right\}$, where the TEM bias is given by

$$
\begin{aligned}
\delta_{\hat{\epsilon}_{(4)}}= & \|\mathbf{H}\|^{-2} 2 \rho_{2} \sum_{j=1}^{N_{r}}\left[2\left(g_{-2}^{\epsilon}-g_{2}^{\epsilon}\right) \Re\left(h_{j 3}^{*} h_{j 2}-h_{j 4}^{*} h_{j 1}\right)\right. \\
& \left.-\left(g_{-1}^{\epsilon}-g_{1}^{\epsilon}-g_{-3}^{\epsilon}+g_{3}^{\epsilon}\right) \Re\left(h_{j 3}^{*} h_{j 1}+h_{j 4}^{*} h_{j 2}\right)\right] .
\end{aligned}
$$

Due to the non-zero bias $\delta_{\hat{\epsilon}_{(4)}}$, the TED in (40) is quasi-robust. Similar to the case of (34) the variance for the TED in (40) can be solved using (16) and (31), where

$$
\boldsymbol{\Sigma}_{\boldsymbol{\Phi}}=4\left(\boldsymbol{\Phi}_{1100}^{R R}+\boldsymbol{\Phi}_{0011}^{R R}-2 \boldsymbol{\Phi}_{1010}^{R R}\right)
$$

and

$$
\boldsymbol{\Sigma}_{\boldsymbol{\Delta}}=4\left(\boldsymbol{\Delta}_{1100}^{R R}+\boldsymbol{\Delta}_{0011}^{R R}\right) .
$$

\section{Properties of Examples of TEDs}

Figs. 2 and 3 show the S-curve for $\hat{\epsilon}_{(2)}$ and $\hat{\epsilon}_{(4)}$ given by (36) and (41), respectively. In the case of $\hat{\epsilon}_{(4)}$, the expectation of the bias $\delta_{\hat{\epsilon}_{(4)}}$ over $\mathbf{H}$ was computed numerically by averaging over $10^{4}$ channel instances. In addition, we verify the theoretical curves via simulation, where the data was sampled at a fixed offset with the timing loop disabled and the TED output $\hat{\epsilon}$ averaged over all code blocks transmitted. Finally, the effect of data decision errors was evaluated by replacing the data symbols in (34) and (40) by their corresponding data decisions for system SNR set to $10 \mathrm{~dB}$ and $20 \mathrm{~dB}$. We note that in

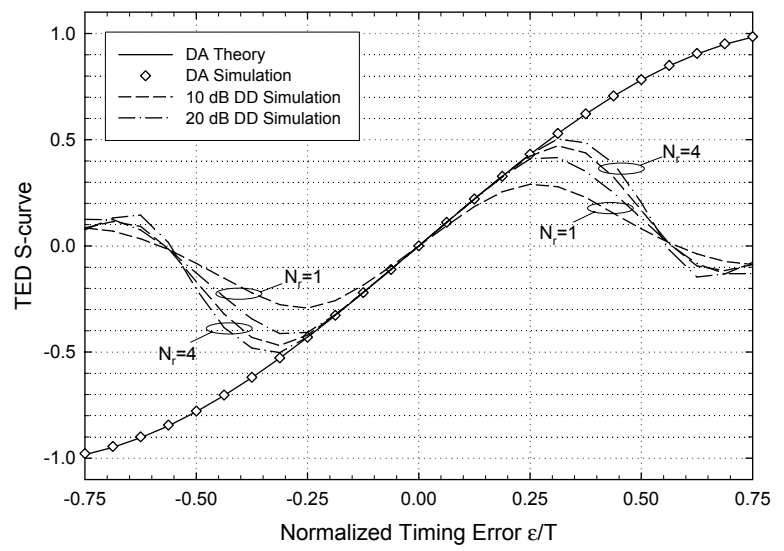

Fig. 2. S-curve for TED $\hat{\epsilon}_{(2)}$ defined in (34); $N_{t}=2, N_{r}=1$ and $N_{r}=4$.

the case of data-aided TED, the simulated results follow the 


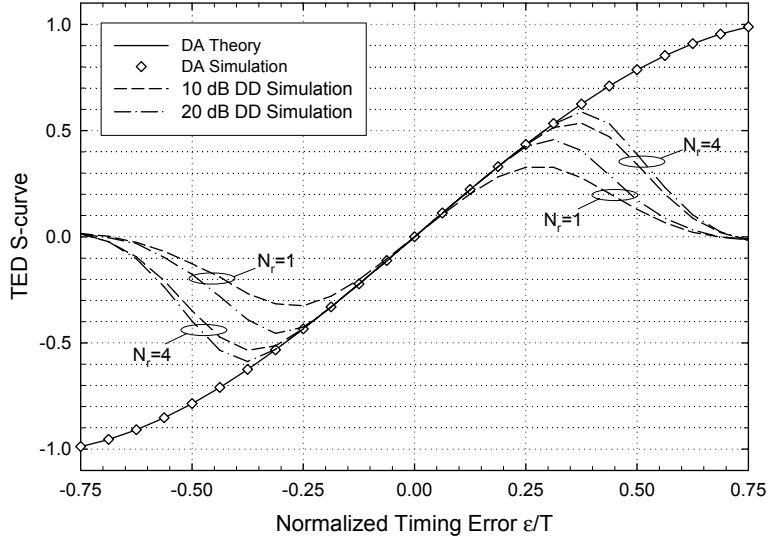

Fig. 3. S-curve for TED $\hat{\epsilon}_{(4)}$ defined in (40); $N_{t}=4, N_{r}=1$ and $N_{r}=4$.

theoretical expressions very closely. Examining the decisiondirected S-curve, we note that incorrect data decisions reduce the linear estimation region to approximately $|\epsilon| / T \approx 0.25$ for SNR of $20 \mathrm{~dB}$, with the range extended to $|\epsilon| / T \approx 0.35$ with increased diversity order.

Figs. 4 and 5 present the TED SNR for $\hat{\epsilon}_{(2)}$ and $\hat{\epsilon}_{(4)}$ with $N_{r}=4$. The theoretical curves were obtained using (32), (31) and (16) with (37), (38) and (43), (44), respectively. The averaging over $\mathbf{H}$ was carried out numerically. The theoretical plots were confirmed by means of simulations using (33) and (16), where the variance was computed by averaging $(\epsilon-\hat{\epsilon})^{2}$ over all data blocks transmitted for a fixed $\epsilon$, open timing loop and system SNR of $10 \mathrm{~dB}$ and $20 \mathrm{~dB}$.

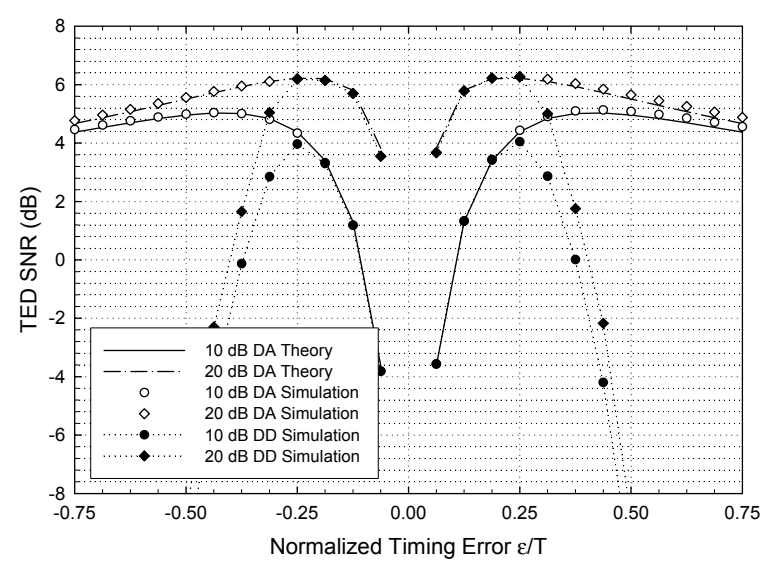

Fig. 4. SNR for TED defined in (34); $N_{t}=2, N_{r}=4$.

For $\epsilon=0$, where no correction is needed, $E\{\hat{\epsilon}\}=0$ and thus TED SNR is zero. For moderate system SNR, the TED SNR peaks at a normalized timing error of below $\epsilon / T=0.25$, which is the usual timing loop operating region. For large timing offset, the estimation variance is large, reducing the TED SNR. This suggest that the loop filter bandwidth should be reduced for low SNR. Examining the decision-directed results, one notes that the errors in data decisions result in a significant reduction of TED SNR, with the TED SNR drop corresponding to the non-linear S-curve region.

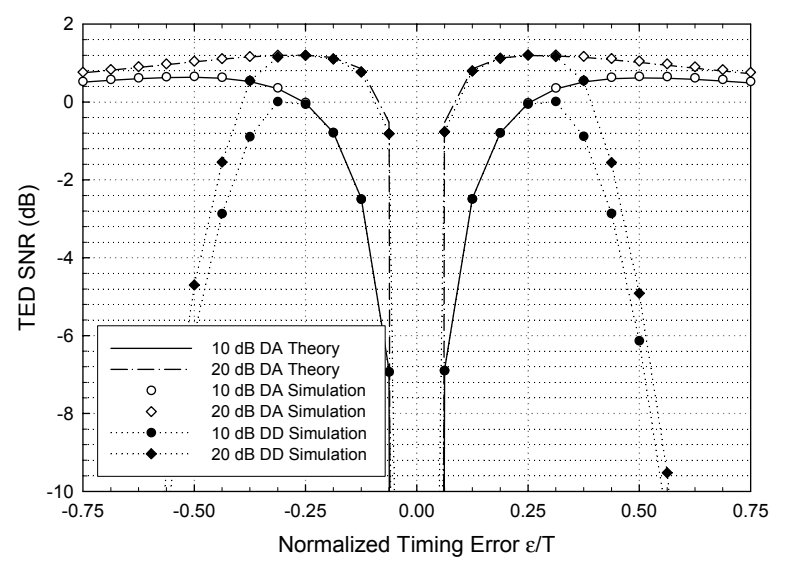

Fig. 5. SNR for TED defined in (40); $N_{t}=4, N_{r}=4$.

\section{Simulation Results}

We present simulation results for OSTBC given by (39) with timing estimation using $\hat{\epsilon}_{(4)}$ in $(40)^{2}$. The data was filtered by a RRC filter with a rolloff of $\beta_{M F}=0.35$. We consider Rayleigh fading with $f_{D} T=0.01$. It is assumed that the receiver has performed coarse timing acquisition, which would typically be done via a training sequence. The timing drift was simulated by perturbing the sampling phase $\tau_{l}$. In order to add a random component to the timing drift, the interval between timing slips, measured in symbol intervals and denoted by $N_{\tau}$, was modeled by a Gaussian random variable, with a mean of $\bar{N}_{\tau}$ and a variance $\sigma_{N_{\tau}}^{2}=0.1 \bar{N}_{\tau}$. The drift direction was random and equiprobable, and the step size fixed to $T / 16$. The mean normalized timing error bandwidth, given by

$$
\bar{B}_{\tau} T=\frac{T / 16}{\bar{N}_{\tau} T}=\frac{1}{16 \bar{N}_{\tau}},
$$

was set to ${ }^{3} \bar{B}_{\tau} T=10^{-4}$. Timing estimation was done using the TED given by (40). Since the focus of the investigation is the tracking performance of the detector, the timing estimation was done without the data knowledge at the receiver. Hence the data symbols $a_{m}$ in (40) were replaced by their estimates $\hat{a}_{m}$. The timing error estimate for code block $l$, that is $\hat{\epsilon}_{l}$, was passed through a first-order IIR filter with the output of

$$
\hat{\epsilon}_{l}^{\prime}=\alpha \hat{\epsilon}_{l-1}^{\prime}+(1-\alpha) \hat{\epsilon}_{l},
$$

where the loop constant $\alpha=0.9$. When $\hat{\epsilon}_{l}^{\prime}$ exceeded a threshold value $\epsilon_{t h}=0.25$, the timing correction $\hat{\tau}_{l}$ was adjusted by a fraction of the symbol interval $T / 8$, depending on the polarity of the error estimate. In practice, this can be implemented using a bank of polyphase filters [11].

In addition to the receiver with perfect channel knowledge, we evaluate the effects of channel estimation errors for a pilot symbol assisted modulation (PSAM) receiver, as described in

\footnotetext{
${ }^{2}$ For detailed description of the simulation setup as well as additional results, the reader is referred to [4], [9].

${ }^{3}$ Current state of the art temperature compensated crystal oscillators (TCXOs) have a frequency stability of well under $10 \mathrm{ppm}$, corresponding to $\bar{B}_{\tau} T<10^{-5}[10]$.
} 
[1]. The data was divided into frames consisting of known orthogonal pilot blocks, followed by 4 OSTBC data code blocks. The received sequence was decimated to recover the pilot symbols, which were used to obtain the channel estimates for the pilot slots. These were subsequently interpolated to obtain channel fading values for the data portion of each frame. In the results presented here, Wiener interpolation filter with 9 interpolants was used.

Figure (6) presents SER performance results for CSIR and PSAM receiver, in addition to two reference curves, namely perfect channel and timing estimation, and perfect timing with PSAM channel estimation. The results demonstrate that the re-

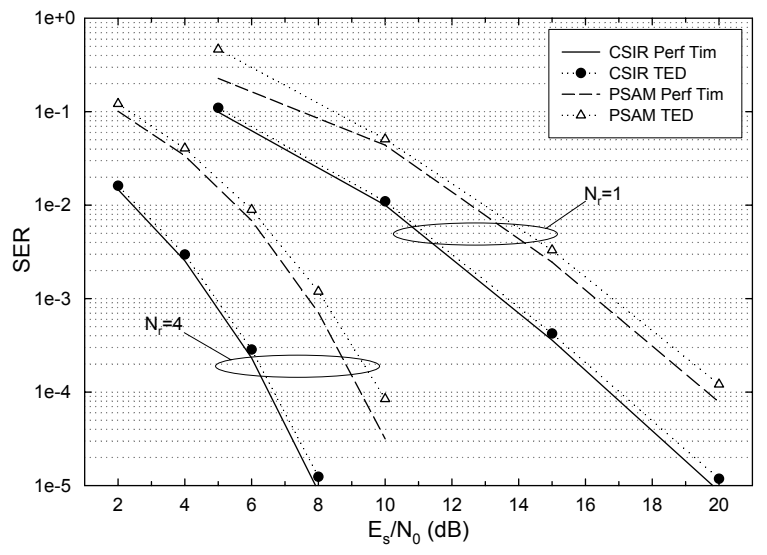

Fig. 6. QPSK SER for $N_{t}=4$ OSTBC in (39).

ceiver is able to track the timing variation with a performance drop of approximately $0.3 \mathrm{~dB}$, both in the case of CSIR and PSAM receivers. By examining the reference curves, we note that for a PSAM receiver with timing error tracking, the SER degradation is dominated by channel estimation errors, with a small loss due to timing synchronization.

\section{CONClusion}

We have derived the variance and TED SNR for a class of low-complexity TEDs applicable to general orthogonal space-time block coded receivers. Examples for particular codes were given and the results were confirmed by means of simulations, including the effects of decision errors. The SER performance for a receiver employing the proposed TEDs within its timing loop was presented, showing timing estimation loss of approximately $0.3 \mathrm{~dB}$.

\section{APPENDIX A}

We present the detailed derivations of (18), (25) and (28). We begin by considering $E^{\mathbf{H}}\left\{a_{i}^{R} a_{j}^{R} s_{m}^{R} s_{n}^{R}\right\}$, as defined by (17). Using the fact that $\operatorname{tr}(\mathbf{A}) \operatorname{tr}(\mathbf{B})=\operatorname{tr}(\mathbf{A} \otimes \mathbf{B})$, we can express (17) by

$$
\begin{aligned}
E^{\mathbf{H}}\left\{a_{i}^{R} a_{j}^{R} s_{m}^{R} s_{n}^{R}\right\}=\|\mathbf{H}\|^{-4} E^{\mathbf{H}}\left\{a_{i}^{R} a_{j}^{R} \times\right. \\
\left.\operatorname{tr}\left(\mathbf{A}_{m} \Re\left(\mathbf{Y}^{H} \mathbf{H}\right) \otimes \mathbf{A}_{n} \Re\left(\mathbf{Y}^{H} \mathbf{H}\right)\right)\right\} .
\end{aligned}
$$

Using (4) along with the distributive property of the Kronecker product, and assuming mutually independent data and noise, after some manipulation one can obtain

$$
E^{\mathbf{H}}\left\{a_{i}^{R} a_{j}^{R} s_{m}^{R} s_{n}^{R}\right\}=\|\mathbf{H}\|^{-4} E^{\mathbf{H}}\left\{a_{i}^{R} a_{j}^{R} \operatorname{tr}\left(\mathbf{\Psi}_{X}^{R R}+\mathbf{\Psi}_{N}^{R R}\right)\right\},
$$

where $\Psi_{X}^{R R}$ and $\Psi_{N}^{R R}$ are defined by

$$
\begin{aligned}
& \mathbf{\Psi}_{X}^{R R}=\sum_{l} \mathbf{A}_{m} \mathbf{G}_{\epsilon, l}^{H} \Re\left(\mathbf{X}_{l}^{H} \mathbf{H}^{H} \mathbf{H}\right) \otimes \\
& \sum_{l^{\prime}} \mathbf{A}_{n} \mathbf{G}_{\epsilon, l^{\prime}}^{H} \Re\left(\mathbf{X}_{l}^{\prime H} \mathbf{H}^{H} \mathbf{H}\right)
\end{aligned}
$$

and

$$
\mathbf{\Psi}_{N}^{R R}=\mathbf{A}_{m} \Re\left(\mathbf{N}^{H} \mathbf{H}\right) \otimes \mathbf{A}_{n} \Re\left(\mathbf{N}^{H} \mathbf{H}\right) .
$$

Examining (48), we recall that the data blocks are given by (1) and note that the data symbols $a_{i}$ and $a_{j}$ in (47) belong to block $l=0$. Since data symbols are zero mean, the summation in (48) will contribute only the $l=l^{\prime}$ terms, with the remaining components vanishing with the expectation operator. Thus, using the fact that $\mathbf{A B} \otimes \mathbf{C D}=\mathbf{A C} \otimes \mathbf{B D}$, we obtain

$$
\begin{array}{r}
E^{\mathbf{H}}\left\{a_{i}^{R} a_{j}^{R} \operatorname{tr}\left(\boldsymbol{\Phi}_{X}^{R R}\right)\right\}=\operatorname{tr}\left\{\sum_{l}\left(\mathbf{A}_{m} \mathbf{G}_{\epsilon, l}^{H} \otimes \mathbf{A}_{n} \mathbf{G}_{\epsilon, l}^{H}\right) \times\right. \\
\left.E^{\mathbf{H}}\left\{a_{i}^{R} a_{j}^{R} \Re\left(\mathbf{X}_{l}^{H} \mathbf{H}^{H} \mathbf{H}\right) \otimes \Re\left(\mathbf{X}_{l}^{H} \mathbf{H}^{H} \mathbf{H}\right)\right\}\right\},
\end{array}
$$

which can be further expanded to

$$
\begin{aligned}
& E^{\mathbf{H}}\left\{a_{i}^{R} a_{j}^{R} \operatorname{tr}\left(\mathbf{\Psi}_{X}^{R R}\right)\right\}= \\
& \operatorname{tr}\left\{\sum _ { l } ( \mathbf { A } _ { m } \mathbf { G } _ { \epsilon , l } ^ { H } \otimes \mathbf { A } _ { n } \mathbf { G } _ { \epsilon , l } ^ { H } ) E ^ { \mathbf { H } } \left\{a_{i}^{R} a_{j}^{R} \times\right.\right. \\
& {\left[\left(\Re\left(\mathbf{X}_{l}^{H}\right) \otimes \Re\left(\mathbf{X}_{l}^{H}\right)\right) \boldsymbol{\Omega}_{\mathrm{RR}}+\left(\Im\left(\mathbf{X}_{l}^{H}\right) \otimes \Im\left(\mathbf{X}_{l}^{H}\right)\right) \boldsymbol{\Omega}_{\mathrm{II}}\right.} \\
&\left.\left.\left.-\left(\Re\left(\mathbf{X}_{l}^{H}\right) \otimes \Im\left(\mathbf{X}_{l}^{H}\right)\right) \boldsymbol{\Omega}_{\mathrm{RI}}-\left(\Im\left(\mathbf{X}_{l}^{H}\right) \otimes \Re\left(\mathbf{X}_{l}^{H}\right)\right) \boldsymbol{\Omega}_{\mathrm{IR}}\right]\right\}\right\},
\end{aligned}
$$

with $\boldsymbol{\Omega}_{\mathrm{RR}}, \boldsymbol{\Omega}_{\mathrm{II}}, \boldsymbol{\Omega}_{\mathrm{RI}}, \Omega_{\mathrm{IR}}$ defined by (23). Using (1), we expand (51), giving

$$
\begin{gathered}
E^{\mathbf{H}}\left\{a_{i}^{R} a_{j}^{R} \operatorname{tr}\left(\mathbf{\Psi}_{X}^{R R}\right)\right\}=\operatorname{tr}\left\{\sum_{l}\left(\mathbf{A}_{m} \mathbf{G}_{\epsilon, l}^{H} \otimes \mathbf{A}_{n} \mathbf{G}_{\epsilon, l}^{H}\right) \times\right. \\
E^{\mathbf{H}}\left\{a_{i}^{R} a_{j}^{R}\left(\sum_{k=0}^{N_{s}-1} a_{k, l}^{R} \mathbf{A}_{k}^{H} \otimes \sum_{k^{\prime}=0}^{N_{s}-1} a_{k^{\prime}, l}^{R} \mathbf{A}_{k^{\prime}}^{H}\right) \boldsymbol{\Omega}_{\mathrm{RR}}\right. \\
+a_{i}^{R} a_{j}^{R}\left(\sum_{k=0}^{N_{s}-1} a_{k, l}^{I} \mathbf{B}_{k}^{H} \otimes \sum_{k^{\prime}=0}^{N_{s}-1} a_{k^{\prime}, l}^{I} \mathbf{B}_{k^{\prime}}^{H}\right) \boldsymbol{\Omega}_{\mathrm{II}} \\
+a_{i}^{R} a_{j}^{R}\left(\sum_{k=0}^{N_{s}-1} a_{k, l}^{R} \mathbf{A}_{k}^{H} \otimes \sum_{k^{\prime}=0}^{N_{s}-1} a_{k^{\prime}, l}^{I} \mathbf{B}_{k^{\prime}}^{H}\right) \boldsymbol{\Omega}_{\mathrm{RI}} \\
\left.\left.+a_{i}^{R} a_{j}^{R}\left(\sum_{k=0}^{N_{s}-1} a_{k, l}^{I} \mathbf{B}_{k}^{H} \otimes \sum_{k^{\prime}=0}^{N_{s}-1} a_{k^{\prime}, l}^{R} \mathbf{A}_{k^{\prime}}^{H}\right) \boldsymbol{\Omega}_{\mathrm{IR}}\right\}\right\} .
\end{gathered}
$$

In (52), we distinguish cases where $i=j$ and $i \neq j$. For $i \neq j$ we note that only $l=0$ with $k=i, k^{\prime}=j$ and $k=j, k^{\prime}=i$ terms will remain. Assuming independent and zero mean date, 
one can show that, for $i \neq j$, the terms involving $\boldsymbol{\Omega}_{\mathrm{RI}}$ and $\boldsymbol{\Omega}_{\mathrm{IR}}$ will vanish with the expectation operator, giving

$$
\begin{array}{r}
E^{\mathbf{H}}\left\{a_{i}^{R} a_{j}^{R} \operatorname{tr}\left(\mathbf{\Psi}_{X}^{R R}\right)\right\}=\rho_{2}^{2} \operatorname{tr}\left\{\left(\mathbf{A}_{m} \mathbf{G}_{\epsilon, 0}^{H} \otimes \mathbf{A}_{n} \mathbf{G}_{\epsilon, 0}^{H}\right) \times\right. \\
\left.\left(\mathbf{A}_{j}^{H} \otimes \mathbf{A}_{i}^{H}+\mathbf{A}_{i}^{H} \otimes \mathbf{A}_{j}^{H}\right) \boldsymbol{\Omega}_{\mathrm{RR}}\right\},
\end{array}
$$

where $\rho_{2}$ is defined by (10). In the case when $i=j$, only terms for $k=k^{\prime}$ in (52) will remain for all $l$. Thus, after some manipulation, one can show that for $i=j$

$$
\begin{aligned}
& E^{\mathbf{H}}\left\{\left(a_{i}^{R}\right)^{2} \operatorname{tr}\left(\mathbf{\Psi}_{X}^{R R}\right)\right\}=\rho_{2}^{2} \operatorname{tr}\left\{\sum_{l} \sum_{k}\left(\mathbf{A}_{m} \mathbf{G}_{\epsilon, l}^{H} \otimes \mathbf{A}_{n} \mathbf{G}_{\epsilon, l}^{H}\right)\right. \\
& \left.\times\left[\left(\mathbf{A}_{k}^{H} \otimes \mathbf{A}_{k}^{H}\right) \boldsymbol{\Omega}_{\mathrm{RR}}+\left(\mathbf{B}_{k}^{H} \otimes \mathbf{B}_{k}^{H}\right) \boldsymbol{\Omega}_{\mathrm{II}}\right]\right\} \\
& +\operatorname{tr}\left\{\left(\mathbf{A}_{m} \mathbf{G}_{\epsilon, 0}^{H} \otimes \mathbf{A}_{n} \mathbf{G}_{\epsilon, 0}^{H}\right)\right. \\
& \left.\times\left[\left(\rho_{4}-\rho_{2}^{2}\right)\left(\mathbf{A}_{i}^{H} \otimes \mathbf{A}_{i}^{H}\right) \boldsymbol{\Omega}_{\mathrm{RR}}+\left(\rho_{2}^{\prime}-\rho_{2}^{2}\right)\left(\mathbf{B}_{i}^{H} \otimes \mathbf{B}_{i}^{H}\right) \boldsymbol{\Omega}_{\mathrm{II}}\right]\right\}
\end{aligned}
$$

where $\rho_{2}$ and $\rho_{4}$ are defined by (10) and $\rho_{2}^{\prime}$ by (22). We note that the first summation accounts for ISI components over all data blocks and will converge since $\mathbf{G}_{\epsilon, l} \rightarrow 0$ as $l \rightarrow \infty$.

Having solved for the data component in (47), we now solve for the noise term, starting with (49). Since $E\left\{a_{i}^{R}\right\}=0$, $E^{\mathbf{H}}\left\{a_{i}^{R} a_{j}^{R} \operatorname{tr}\left(\mathbf{\Psi}_{N}^{R R}\right)\right\}$ will be non-zero only for $i=j$. In such a case, we can write

$$
\begin{aligned}
& E^{\mathbf{H}}\left\{\left(a_{i}^{R}\right)^{2} \operatorname{tr}\left(\mathbf{\Psi}_{N}^{R R}\right)\right\}=\rho_{2} \operatorname{tr}\left\{\left(\mathbf{A}_{m} \otimes \mathbf{A}_{n}\right) \times\right. \\
& E^{\mathbf{H}}\left\{\left(\Re\left(\mathbf{N}^{H}\right) \otimes \Re\left(\mathbf{N}^{H}\right)\right) \boldsymbol{\Omega}_{\mathrm{RR}}^{\prime}+\left(\Im\left(\mathbf{N}^{H}\right) \otimes \Im\left(\mathbf{N}^{H}\right)\right) \boldsymbol{\Omega}_{\mathrm{II}}^{\prime}\right. \\
& \left.\left.\quad-\left(\Re\left(\mathbf{N}^{H}\right) \otimes \Im\left(\mathbf{N}^{H}\right)\right) \boldsymbol{\Omega}_{\mathrm{RI}}^{\prime}-\left(\Im\left(\mathbf{N}^{H}\right) \otimes \Re\left(\mathbf{N}^{H}\right)\right) \boldsymbol{\Omega}_{\mathrm{IR}}^{\prime}\right\}\right\},
\end{aligned}
$$

where $\boldsymbol{\Omega}_{\mathrm{RR}}^{\prime}, \boldsymbol{\Omega}_{\mathrm{II}}^{\prime}, \boldsymbol{\Omega}_{\mathrm{RI}}^{\prime}, \boldsymbol{\Omega}_{\mathrm{IR}}^{\prime}$ are defined (24). Since the real and imaginary noise components are assumed independent and zero-mean, the last two terms in (55) will vanish, giving

$$
E^{\mathbf{H}}\left\{\left(a_{i}^{R}\right)^{2} \operatorname{tr}\left(\mathbf{\Psi}_{N}^{R R}\right)\right\}=\rho_{2} \operatorname{tr}\left\{\left(\mathbf{A}_{m} \otimes \mathbf{A}_{n}\right) \boldsymbol{\Lambda}_{N}\left(\boldsymbol{\Omega}_{\mathrm{RR}}^{\prime}+\boldsymbol{\Omega}_{\mathrm{II}}^{\prime}\right)\right\},
$$

where the matrix $\boldsymbol{\Lambda}_{N}$ is defined by (21). Finally, combining (53), (54) and (56) with (47), and defining $\boldsymbol{\Phi}_{i j m n}^{R R}=$ $a_{i}^{R} a_{j}^{R} \Psi_{X}^{R R}$ and $\boldsymbol{\Delta}_{i j m n}^{R R}=\left(a_{i}^{R}\right)^{2} \boldsymbol{\Psi}_{N}^{R R}$, leads to (18).

The solution to $E^{\mathbf{H}}\left\{a_{i}^{I} a_{j}^{I} s_{m}^{I} s_{n}^{I}\right\}$ can be obtained by following the same methodology as for $E^{\mathbf{H}}\left\{a_{i}^{R} a_{j}^{R} s_{m}^{R} s_{n}^{R}\right\}$ with the solution given by (25).

Finally, we consider $E^{\mathbf{H}}\left\{a_{i}^{R} a_{j}^{I} s_{m}^{R} s_{n}^{I}\right\}$, which, using the same reasoning as for $E^{\mathbf{H}}\left\{a_{i}^{R} a_{j}^{R} s_{m}^{R} s_{n}^{R}\right\}$, can be expressed as

$$
E^{\mathbf{H}}\left\{a_{i}^{R} a_{j}^{I} s_{m}^{R} s_{n}^{I}\right\}=-\|\mathbf{H}\|^{-4} E^{\mathbf{H}}\left\{a_{i}^{R} a_{j}^{I} \operatorname{tr}\left(\mathbf{\Psi}_{X}^{R I}+\mathbf{\Psi}_{N}^{R I}\right)\right\},
$$

where

$$
\begin{array}{r}
\mathbf{\Psi}_{X}^{R I}=\sum_{l} \mathbf{A}_{m} \mathbf{G}_{\epsilon, l}^{H} \Re\left(\mathbf{X}_{l}^{H} \mathbf{H}^{H} \mathbf{H}\right) \otimes \\
\sum_{l^{\prime}} \mathbf{B}_{n} \mathbf{G}_{\epsilon, l^{\prime}}^{H} \Im\left(\mathbf{X}_{l}^{\prime H} \mathbf{H}^{H} \mathbf{H}\right)
\end{array}
$$

and

$$
\mathbf{\Psi}_{N}^{R I}=\mathbf{A}_{m} \Re\left(\mathbf{N}^{H} \mathbf{H}\right) \otimes \mathbf{B}_{n} \Im\left(\mathbf{N}^{H} \mathbf{H}\right) .
$$

We note that, since the real and imaginary components of $\mathbf{N}$ are independent, $E^{\mathbf{H}}\left\{a_{i}^{I} a_{i}^{I} \operatorname{tr}\left(\mathbf{\Psi}_{N}^{R I}\right)\right\}=0$ and thus we need only consider the first term of (57). One can show that

$$
\begin{aligned}
& E^{\mathbf{H}}\left\{a_{i}^{R} a_{j}^{I} \operatorname{tr}\left(\mathbf{\Psi}_{X}^{R I}\right)\right\}= \\
& \operatorname{tr}\left\{\sum _ { l } ( \mathbf { A } _ { m } \mathbf { G } _ { \epsilon , l } ^ { H } \otimes \mathbf { A } _ { n } \mathbf { G } _ { \epsilon , l } ^ { H } ) E ^ { \mathbf { H } } \left\{a_{i}^{R} a_{j}^{I} \times\right.\right. \\
& {\left[-\left(\Re\left(\mathbf{X}_{l}^{H}\right) \otimes \Im\left(\mathbf{X}_{l}^{H}\right)\right) \boldsymbol{\Omega}_{\mathrm{RR}}+\left(\Im\left(\mathbf{X}_{l}^{H}\right) \otimes \Re\left(\mathbf{X}_{l}^{H}\right)\right) \boldsymbol{\Omega}_{\mathrm{II}}\right.} \\
& \left.\left.\quad-\left(\Re\left(\mathbf{X}_{l}^{H}\right) \otimes \Re\left(\mathbf{X}_{l}^{H}\right)\right) \boldsymbol{\Omega}_{\mathrm{RI}}+\left(\Im\left(\mathbf{X}_{l}^{H}\right) \otimes \Im\left(\mathbf{X}_{l}^{H}\right)\right) \boldsymbol{\Omega}_{\mathrm{IR}}\right]\right\},
\end{aligned}
$$

where the terms involving $\Omega_{\mathrm{RI}}$ and $\boldsymbol{\Omega}_{\mathrm{IR}}$ will once again vanish with the expectation operator. For the same reason, we note that for all $i$ and $j$, only the summation term $l=0$ will survive, resulting in

$$
\begin{gathered}
E^{\mathbf{H}}\left\{a_{i}^{R} a_{j}^{I} \operatorname{tr}\left(\mathbf{\Psi}_{X}^{R I}\right)\right\}=\operatorname{tr}\left\{\left(\mathbf{A}_{m} \mathbf{G}_{\epsilon, 0}^{H} \otimes \mathbf{B}_{n} \mathbf{G}_{\epsilon, 0}^{H}\right) \times\right. \\
E^{\mathbf{H}}\left\{a_{i}^{R} a_{j}^{I}\left(\sum_{k} a_{k, l}^{R} \mathbf{A}_{k}^{H} \otimes \sum_{k^{\prime}} a_{k^{\prime}, l}^{R} \mathbf{B}_{k^{\prime}}^{H}\right) \boldsymbol{\Omega}_{\mathrm{RR}}\right. \\
\left.\left.-a_{i}^{R} a_{j}^{I}\left(\sum_{k^{\prime}} a_{k^{\prime}, l}^{I} \mathbf{B}_{k^{\prime}}^{H} \otimes \sum_{k} a_{k, l}^{I} \mathbf{A}_{k}^{H}\right) \boldsymbol{\Omega}_{\mathrm{II}}\right\}\right\} .
\end{gathered}
$$

Examining (61), we see that non-zero terms will involve $k=i$ and $k^{\prime}=j$, and thus defining $\boldsymbol{\Phi}_{i j m n}^{R I}=a_{i}^{R} a_{j}^{I} \boldsymbol{\Psi}_{X}^{R I}$, one obtains the solution given by (28).

\section{ACKNOWLEDGMENT}

The authors would like to thank NSERC of Canada and the Bell Mobility / Samsung Grant on Smart Antennas at Queen's University for their support of this research, as well as an anonymous reviewer for the input on the definition of TED SNR.

\section{REFERENCES}

[1] A.F.Naguib, V.Tarokh, N.Seshadri, and R.Calderbank. A space-time coding modem for high-data-rate wireless communications. IEEE J. Select. Areas Commun., 16(8):1459-1478, October 1998.

[2] Y.-C.Wu, S.C.Chan, and E.Serpedin. Symbol-timing estimation in spacetime coding systems based on orthogonal training sequences. IEEE Trans. Wireless Commun., 4(2):603-613, March 2005.

[3] K.Rajawat and A.K.Chaturvedi. A low complexity symbol timing estimator for MIMO systems using two samples per symbol. IEEE Communications Letters, 10(7):525-527, July 2006.

[4] P.A.Dmochowski and P.J.McLane. Timing error detector design for orthogonal space-time block code receivers. In IEEE PIMRC 2006, September 2006.

[5] G.Ganesan and P.Stoica. Space-time block codes: A maximum SNR approach. IEEE Trans Inf. Theory, 47(4):1650-1656, May 2001.

[6] P.A.Dmochowski and P.J.McLane. Robust timing epoch tracking for Alamouti space-time coding in flat Rayleigh fading MIMO channels. In Proc. IEEE International Conference on Communications (ICC), pages 2397-2401, May 2005.

[7] E.G.Larsson and P.Stoica. Space-Time Block Coding for Wireless Communications. Cambridge University Press, 2003.

[8] B.Vucetic and J.Yuan. Space-Time Coding. John Wiley \& Sons Canada Ltd, 2003.

[9] P.A. Dmochowski. Design and Analysis of Pulse-Shaped Space-Time Block Code Based Symbol Timing Recovery Algorithms. PhD thesis, Queen's University, Kingston, ON, Canada, September 2006.

[10] W.Zhou, H.Zhou, Z.Xuan, and W.Zhang. Comparison among precision temperature compensated crystal oscillators. In Proc. IEEE International Frequency Control Symposium and Exposition, pages 575-579, August 2005.

[11] F.J.Harris and M.Rice. Multirate digital filters for symbol timing synchronization in software defined radios. IEEE Journal on Selected Areas in Communications, 19(12):2346-2357, December 2001. 